\title{
Efeitos da Fisioterapia com Trampolim no Traumatismo Raquimedular: Estudo de Caso
}

\author{
Physical Therapy on the Trampoline Effects in Spinal Cord Injury: Case Report
}

Eloisa Mombelli Citero ${ }^{1}$, Eduardo Mário Mederdrut ${ }^{2}$, Sissy Veloso Fontes ${ }^{3}$

\begin{abstract}
RESUMO
Introdução. A lesão medular é uma desordem neurológica frequente, importante causa de morbidade e mortalidade e, que traz consequências graves, às vezes irreversíveis, na vida dos indivíduos acometidos. $\mathrm{O}$ trampolim é um recurso fisioterapêutico que pode contribuir para a recuperação de pacientes com acometimento neurológico. Objetivo. Verificar os efeitos da intervenção fisioterapêutica (cinesioterapia) utilizando o trampolim no equilíbrio, na marcha, na flexibilidade articular e no tono muscular de uma paciente com trauma raquimedular incompleto, nível C7-T1, imediatamente após o término do tratamento e após seis meses sem intervenção. Método. Foi realizada avaliação do equilíbrio (escala de Berg), marcha (escala de Tinetti), tono (escala de Ashworth) e flexibilidade (flexímetro) pré, pós-tratamento e, imediatamente seis meses depois do término do tratamento, sem intervenção. Resultados. Obteve-se melhora do equilíbrio e da marcha, houve interferência no tono muscular, na flexibilidade articular ora positiva, ora negativamente dependendo da articulação analisada, depois da intervençáo e no follow up. Conclusáo. cinesioterapia com trampolim melhorou o equilíbrio e a marcha e, modificou o tono e a flexibilidade articular, porém a manutenção desses efeitos após seis meses sem tratamento foi heterogenia em um caso de lesão medular traumática incompleta.
\end{abstract}

Unitermos. Traumatismos da Medula Espinal, Fisioterapia (Especialidade), Equilíbrio, Marcha, Tono Muscular.

Citaçáo. Citero EM, Mederdrut EM, Fontes SV. Efeitos da Fisioterapia com Trampolim no Traumatismo Raquimedular: Estudo de Caso.

\begin{abstract}
Introduction. The spinal cord injury is a common neurologic disorder and an important cause of morbity and mortality in current society. Yours consequences bring severe and either irreversible changes, in individual's life attacked by this injury. The trampoline is more one physiotherapeutic resource that come to contribute for treatment of this and other similar cases. Objective. Check the effect of physiotherapy intervention using the trampoline in the improvement of balance and consequently on the gait as well as verifying the influence in articulation flexibility and in the spasticity of a patient with incomplete spinal cord injury, level C7-T1, immediately after the end of the treatment and after six month. Method. A valuation of balance, gait, spasticity and flexibility was achieved before, after treatment and immediately six month after the finish treatment, using the respective scales. Results. in accordance with the assessment, obtained an improvement of balance, gait, it had an interference in the spasticity, in the articulation flexibility, sometimes positive or negatively subject of articulation analysed, after intervention and on follow up. Conclusion. the trampoline was efficient in the improvement of balance and gait, and influenced spasticity and flexibility, but in a heterogeneous way.
\end{abstract}

Keywords. Spinal Cord Injuries, Physiotherapy, Balance, Gait, Muscle Tonus.

Citation. Citero EM, Mederdrut EM, Fontes SV. Physical Therapy on the Trampoline Effects in Spinal Cord Injury: Case Report.
Pesquisa realizada na Universidade Metodista de São Paulo (Umesp), São Paulo-SP, Brasil.

1. Fisioterapeuta, Especialista em Fisioterapia Neurológica pela Umesp, São Paulo-SP, Brasil.

2. Fisioterapeuta, Especialista em Fisioterapia Esportiva, São Paulo-SP, Brasil.

3. Fisioterapeuta e Pesquisadora do Departamento de Neurologia e Neurocirurgia da Unifesp, Professora de Educaçáo Física, Doutora em Ciências pela Unifesp e Coordenadora do Curso de Especializaçấo em Teorias e Técnicas para Cuidados Integrativos da Unifesp, Sáo Paulo-SP, Brasil.
Endereço para correspondência: Sissy V. Fontes R Francisco Tapajós, 513 apto.122 - Vila Santo Estéfano CEP 04153-001, São Paulo-SP, Brasil. E-mail: sissyfontes@gmail.com

Relato de Caso Recebido em: 05/06/06 Aceito em: 11/08/09 Conflito de interesses: não 


\section{INTRODUÇÃO}

A lesão medular é uma agressão à medula espinal resultando em mudanças temporárias ou permanentes, da função motora, sensitiva, autonômica normal ${ }^{1,2}$. O tipo de traumatismo raquimedular (TRM) e sua localização determinam a natureza e a extensão da lesão, podendo ocasionar diversas disfunçôes, dentre elas a alteração do equilíbrio que, consequentemente influencia a marcha, alteração do tono muscular e da flexibilidade articular, que interferem de maneira negativa as atividades funcionais e a qualidade de vida de indivíduos com TRM ${ }^{3}$.

Essa desordem neurológica é uma importante causa de morbidade e mortalidade em todo o mundo. No Brasil há poucos dados e trabalhos publicados a respeito da epidemiologia da lesão medular. Estima-se que ocorram a cada ano, no Brasil, mais de 10.000 novos casos de lesão medular ${ }^{4}$.

A fisioterapia exerce um papel fundamental na assistência imediata e tardia do paciente, estimulando a recuperação funcional de modo a propiciar maior independência possível, e engloba vários procedimentos, dentre eles, a mecanoterapia que consiste em exercícios físicos e ou atividades terapêuticas realizadas com auxílio de instrumentos ${ }^{5}$, entre esses instrumentos inclui-se o trampolim, que é um novo recurso que está sendo utilizado na fisioterapia neurofuncional ${ }^{6}$.

O trampolim acrobático é popularmente conhecido como cama elástica, e desde a idade média, já era utilizado como forma de divertimento para os reis que eram entretidos pelos "bobos da corte". No Brasil, a cama elástica começou a surgir em 1974, quando o campeão mundial desta modalidade na época, o alemão Hartmut Rihle, aqui esteve para ensinar as técnicas acrobáticas e despertou o interesse do professor brasileiro José de Oliveira Martins. A utilização do trampolim como instrumento de tratamento de afecçóes teve seu início quando o fisioterapeuta Eduardo Mario Mederdut o trouxe para o Brasil para ser utilizado na recuperação de lesôes músculo-esqueléticas de atletas 5 .

Em 2003, teve início na Clínica Escola de Fisioterapia da Universidade Metodista de São Paulo (Umesp), um grupo de pesquisa coordenado pela Profa. Dra. Sissy Veloso Fontes, no qual se investigou os efeitos do trampolim em pacientes adultos com disfunções neurológicas. $\mathrm{O}$ primeiro trabalho foi realizado por Otsuka, que avaliou os efeitos da cinesioterapia com o trampolim em um caso de paciente atáxico por lesão ponto-cerebelar ${ }^{6}$.

Para a capacitação técnica dos pesquisadores (alunos do último semestre do Curso de Graduação em Fisioterapia da Umesp), na prática de exercícios físicos terapêuticos sobre o trampolim, foram realizados cursos de capacitação em "Trampolim Terapêutico" - Módulo Neurológico, idealizado e organizado pela Profa. Dra. Sissy Veloso Fontes, ministrado pelo fisioterapeuta Eduardo Mário Merdedrut, no Centro de Performance Humana em São Paulo, capital em março de 2004 (1º curso) e em março de 2005 (2º curso). Esses cursos contaram com a participação voluntária de pacientes neurológicos adultos com diferentes tipos de doenças para investigaçáo em oficinas práticas, visando discutir os efeitos fisiológicos observados pelo grupo de estudo, do novo método de tratamento e fundamentação teórica da prática clínica, incluindo medidas de segurança.

Após a capacitação técnica do grupo, em 2004, outros estudos (trabalhos de conclusão de curso) foram realizados, pelo mesmo grupo de pesquisa na Umesp, investigando os efeitos deste recurso em pacientes com outros processos neurológicos: acidente vascular cerebral, doença de Parkinson, esclerose múltipla e paralisia cerebral; sendo observados efeitos positivos quanto à recuperação do equilíbrio, devido, provavelmente à hiper-estimulação proprioceptiva, vestibular e visual que os exercícios terapêuticos realizados sobre a superfície instável do trampolim promoveram, de maneira a facilitar ajustes posturais, assim como, uma melhora na velocidade da marcha, podendo interferir no tono muscular e na flexibilidade articular de membros inferiores, principalmente ${ }^{7-10}$.

É importante salientar que, o uso do trampolim deve ser realizado por fisioterapeutas treinados, pois este procedimento quando realizado de maneira inapropriada pode causar acidentes do tipo fraturas, lacerações, lesóes neurológicas e danos vasculares, portanto, a formação do profissional, nesse método de intervenção fisioterapêutica deve ser realizada em cursos de extensão especializados ${ }^{11}$.

Sendo assim, este trabalho tem como objetivo verificar os efeitos da cinesioterapia utilizando como recurso o trampolim no equilíbrio, na marcha, no tono muscular e na flexibilidade articular de paciente com 
traumatismo raquimedular incompleto nível C7-T1.

\section{MÉTODO}

\section{Caso clínico}

Este trabalho relata um estudo de caso de paciente M.T.P. de 35 anos, branca, casada, artista plástica que apresenta quadro sindrômico de tetraparesia espástica, com alteração de equilíbrio, marcha independente com uso de dispositivo auxiliar (muletas canadense), realizado na Clínica Escola de Fisioterapia da Umesp.

\section{Avaliaçáo}

Foi aplicada uma avaliação de equilíbrio e marcha antes, imediatamente após o tratamento e seis meses após o término do tratamento (follow up), assim como, uma avaliação do tônus muscular e da flexibilidade articular. O follow up consistiu de uma nova reavaliação de equilíbrio, marcha, tono muscular e flexibilidade articular para verificar se houve manutenção ou perda dos resultados alcançados, imediatamente após o término do programa de intervenção fisioterapêutica. Vale lembrar que a paciente ficou aproximadamente seis meses sem qualquer tipo de intervenção fisioterapêutica após o término do tratamento sobre o trampolim.

Os instrumentos de mensuração utilizados foram: escala de equilíbrio de Berg, que utiliza 14 itens graduados de 0 (alteração do equilíbrio) a 4 pontos (equilíbrio normal) com uma pontuação máxima de 56 pontos $^{12-14}$, escala de marcha de Tinetti, que avalia também o equilíbrio e as habilidades em ter mobilidade em adultos mais velhos e sua pontuação varia de 0 (equilíbrio e marcha gravemente prejudicados) a 12 pontos (equilíbrio e marcha normais) ${ }^{12,15,16}$, escala de Ashworth ${ }^{17}$, que mensura a hipertonia muscular durante a mobilizaçáo passiva de determinados grupos musculares, sendo considerado grau 0 (não há aumento no tono), grau 1 (sutil aumento no tono, manifestado por agarramento e soltura, por uma resistência mínima no final do movimento, quando a parte afetada move-se em flexão ou extensão), grau 1+ (sutil aumento no tono manifestado por agarramento, seguido por resistência mínima ao movimento), grau 2 (aumento mais acentuado do tono muscular de um extremo ao outro, mas a parte afetada move-se facilmente), grau 3 (aumento considerável do tono com movimentação pas- siva difícil), grau 4 (parte afetada rígida em extensão ou flexão) e; avaliação da flexibilidade articular com uso do flexímetro $^{18}$. $\mathrm{Na}$ avaliação da flexibilidade articular foram consideradas alteradas as amplitudes de movimento (ADM) que alteraram em 5 ou mais graus de amplitude em todas as articulaçóes avaliadas, ou seja, para ser considerado melhora ou piora da flexibilidade articular, as diferenças das ADM entre as avaliaçôes pré e pós-tratamento deveriam ser maior do que cinco pontos.

\section{Programa de tratamento}

O programa constituiu de 12 (doze) sessōes de 60 minutos cada, com uma frequência de duas vezes por semana, em um período de 3 (três) meses consecutivos de cinesioterapia (exercícios físicos terapêuticos) sobre o trampolim.

Os exercícios propostos tiveram como objetivo estimular capacidades condicionantes e coordenativas ${ }^{19}$, com ênfase nas reaçóes de equilíbrio e proteção em superfície instável, coordenação motora e ritmo e conscientização do corpo no espaço. Inicialmente foram realizados com auxílio do terapeuta progredindo para a realização dos exercícios sem assistência do terapeuta, de acordo com a evolução do paciente nas tarefas. As atividades físicas realizadas respeitaram a tolerância ao exercício do paciente. Dois exemplos de exercícios aplicados são apresentados na Figura 1: exercício que visou estimular a reeducação da marcha. Foi executado com algumas variaçóes e progressóes como: transferência de peso látero-lateral, ântero-posterior, com auxílio da terapeuta e, depois sem o auxílio da terapeuta; exercício visando estimular o controle postural do tronco com transferência de peso sobre os ísquios e pés com ou sem dissociação de cinturas, associado ou não aos desequilíbrios promovidos pela instabilidade da lona do trampolim.

Figura 1. Fisioterapeuta e paciente com TRM sobre o trampolim realizando treino de marcha com apoio anterior e com olhos abertos.

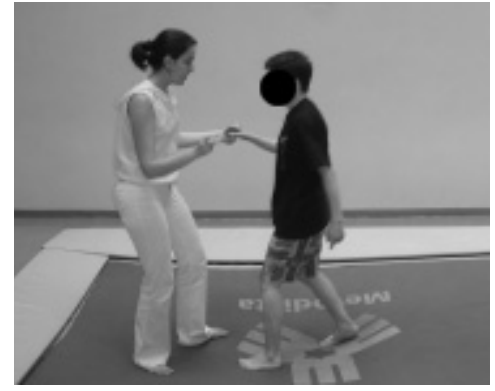




\section{Material}

Foram utilizados os seguintes materiais mecanoterapêuticos: o trampolim, instalado na Clínica Escola da Umesp, que mede 2,8 metros de comprimento por 1,8 metro de largura e está montada sobre uma cavidade com 1,6 metro de profundidade, fabricado por Tramp Brasil; bola suíça da marca Gymnic (made in Italy), com diâmetro de $75 \mathrm{~cm}$; rolo de espuma ("espaguetes") fabricados pela The Toy Power, medindo 1,5m de comprimento, fabricado em espuma, atóxico e inodoro; e outras bolas de diferentes tamanhos e pesos.

\section{Análise dos resultados}

Os resultados são apresentados tabulados, sendo comparados os valores dos escores aos graus de amplitudes de movimento nas avaliações inicial, final e após 6 meses do término do tratamento, sendo consideradas para análise apenas as diferenças numéricas de 1 ou mais pontos para os escores e, 5 ou mais graus para as amplitudes de movimento.

\section{RESULTADOS}

Quanto ao equilíbrio, mensurado com a escala de equilíbrio de Berg observou-se que houve manutenção da maioria dos itens avaliados, melhora na pontuação total entre as avaliaçóes inicial e imediatamente após o tratamento, quanto ao item girando 360 o, passando de 54 para 56 pontos, já no follow-up a paciente regrediu um ponto nos itens 10, 12 e 13, havendo diminuição da pontuação total para 53 pontos, indicando piora em relação ao equilíbrio após 6 meses do término da intervenção fisioterapêutica (Tabela 1).

$\mathrm{Na}$ escala de Tinetti, observou-se melhora em cinco dos sete itens avaliados, entre avaliaçáo inicial e imediatamente após o tratamento, enquanto que no follow up houve piora na maioria dos itens reavaliados (Tabela 2).

Observando os resultados quanto ao tono muscular, em relação ao membro superior direito (MSD) e esquerdo (MSE), um movimento articular piorou, um melhorou e dezesseis mantiveram-se iguais imediatamente após o tratamento, já no follow-up dos movimentos do MSD, dois melhoraram, três pioraram e treze mantiveram-se iguais, enquanto que para o MSE, dois melhoraram e dezesseis permaneceram iguais. Analisando o membro inferior direito (MID), cinco movimentos melhoraram, um piorou e seis mantiveram-se iguais imediatamente após o tratamento e no follow-up, enquanto que para MIE imediatamente após o tratamento, um movimento melhorou, onze permaneceram iguais e, no follow-up, dois melhoraram e dez mantiveram-se iguais (Tabela 3).

Quanto à flexibilidade articular, dos treze movimentos examinados nos membros superiores, no direito (MSD) houve melhora em sete deles, piora em um (desvio radial) e os demais movimentos (cinco) se mantiveram iguais entre as avaliaçóes iniciais e imediatamente após o tratamento, já após seis meses do término da intervençáo (follow up), cinco melhoram, quatro pioraram (flexão e rotação lateral do ombro, flexão do cotovelo e desvio ulnar) e quatro se mantiveram iguais. No esquerdo houve melhora em apenas quatro deles, piora em dois (flexâo de ombro e pronação) e os demais se mantiveram imediatamente após o término do tratamento, no entanto, no follow up, oito melhoraram, quatro pioraram e os demais se mantiveram.

Quanto aos membros inferiores, no direito houve melhora em cinco movimentos e piora em um (abdução do quadril) após o tratamento e, melhora em um (dorsiflexão de tornozelo) e piora em um (flexão de joelho) no follow up; no esquerdo, houve melhora em seis movimentos e piora em um (flexão do quadril) após o tratamento e, melhora em três e piora em um (plantiflexão de tornozelo) no follow up (Tabela 4).

\section{DISCUSSÃO}

Apesar da escala de equilíbrio de Berg não ter sido suficientemente sensível para avaliar o equilíbrio da paciente, pois se obteve quase o escore total na primeira avaliação, isso não significa que não houve melhora dessa capacidade após o tratamento no trampolim, pois, com base nos escores na escala de Tinetti e no relato espontâneo da paciente (esta afirmou que, durante e após o término do tratamento, teve sensação de maior segurança e firmeza ao levantar-se do chão, diminuição de queda da própria altura, maior facilidade em realizar movimentos com o tronco na posição sentada ou em pé, parada ou andando, quando necessitava alcançar objetos, facilitando a realização das atividades de vida diária de autocuidado), 
Tabela 1

Valores obtidos antes, imediatamente após e, após 6 meses (follow up) do tratamento, utilizando como instrumento de mensuração a escala de equilíbrio de Berg e sua pontuação máxima

\begin{tabular}{|c|c|c|c|c|}
\hline Itens da Escala de equilíbrio de Berg & $\begin{array}{c}\text { Avaliaçáo } \\
\text { Pré }\end{array}$ & $\begin{array}{c}\text { Avaliaçáo } \\
\text { Pós }\end{array}$ & Follow $U p$ & $\begin{array}{c}\text { Pontuaçáo } \\
\text { Máxima }\end{array}$ \\
\hline 1. Sentado para em pé & 4 & 4 & 4 & 4 \\
\hline 2. Em pé sem apoio & 4 & 4 & 4 & 4 \\
\hline 3. Sentado sem apoio & 4 & 4 & 4 & 4 \\
\hline 4. Em pé para sentado & 4 & 4 & 4 & 4 \\
\hline 5. Transferências & 4 & 4 & 4 & 4 \\
\hline 6. Em pé com os olhos fechados & 4 & 4 & 4 & 4 \\
\hline 7. Em pé com os pés juntos & 4 & 4 & 4 & 4 \\
\hline 8. Alcance à frente com braços estendidos & 4 & 4 & 4 & 4 \\
\hline 9. Apanhar um objeto do cháo & 4 & 4 & 4 & 4 \\
\hline 10. Virando-se para olhar para trás & 4 & 4 & $3 \#$ & 4 \\
\hline 11. Girando 360o & 2 & $4^{*}$ & 4 & 4 \\
\hline 12. Colocar os pés alternadamente sobre um banco & 4 & 4 & $3 \#$ & 4 \\
\hline 13. Em pé sem apoio com um pé na frente do outro & 4 & 4 & $3 \#$ & 4 \\
\hline 14. Em pé apoiado em uma perna & 4 & 4 & 4 & 4 \\
\hline ESCORE & 54 & 56 & 53 & 56 \\
\hline
\end{tabular}

Os valores com asterisco $\left(^{*}\right)$ indicam que houve alteração positiva e com jogo da velha (\#) negativa entre as avaliaçôes inicial, final e ou follow-up.

Tabela 2

Valores obtidos antes, imediatamente após e, após 6 meses (follow up) do tratamento, utilizando como instrumento de mensuração a escala de marcha de Tinetti e sua pontuação máxima

\begin{tabular}{|c|c|c|c|c|}
\hline Itens da Escala de Tinetti & $\begin{array}{c}\text { Avaliaçáo Pré } \\
\text { tratamento }\end{array}$ & $\begin{array}{c}\text { Avaliaçáo Pós } \\
\text { tratamento }\end{array}$ & Follow $U p$ & $\begin{array}{c}\text { Pontuaçáo } \\
\text { Máxima }\end{array}$ \\
\hline 1. Iniciaçáo da marcha & 1 & 1 & 1 & 1 \\
\hline 2. Comprimento e altura do passo & 0 & $4^{*}$ & 2\# & 4 \\
\hline 3. Simetria do passo & 0 & $1^{*}$ & 1 & 1 \\
\hline 4. Continuidade do passo & 0 & $1^{*}$ & 1 & 1 \\
\hline 5. Desvio da linha reta & 1 & $2^{*}$ & 2 & 2 \\
\hline 6. Tronco & 1 & $2^{*}$ & $1 \#$ & 2 \\
\hline 7. Base de apoio & 0 & 0 & 0 & 1 \\
\hline ESCORE & 3 & 11 & 8 & 12 \\
\hline
\end{tabular}

Os valores com asterisco $\left(^{*}\right)$ indicam que houve alteração positiva e com jogo da velha (\#) negativa entre as avaliaçôes inicial, final e ou follow-up. 
esse programa parece ter interferido positivamente no equilíbrio e na marcha nesse caso, pois a paciente passou a deambular, em algumas ocasióes, sem auxílio da muleta canadense tanto em casa como no trabalho.

No entanto, após seis meses do término do tratamento, na reavaliação da paciente (follow-up), ela relata ter aumentado a frequência de quedas da própria altura, o que pode ser outro indicador de que quando estava recebendo tratamento no trampolim houve melhora de sua estabilidade, mas na ausência desse, os benefícios não se mantiveram. Esses resultados inferem que o programa utilizado, exercícios físicos terapêuticos realizados em superfícies instáveis, trouxe benefícios tanto para a postura estática, como para a dinâmica, mas a prática constante desse deve ser mantida e incentivada.

A hipótese é de que a instabilidade do meio promoveu diferentes graus de dificuldade para realização das diversas atividades físicas orientadas, o que estimulou estratégias sensório-motoras mais refinadas para manter alinhamento, estabilidade do corpo e, permitiu movimentos em posturas mais complexas, além de melhorar a orientação espacial, facilitando, assim ajustes posturais de melhor qualidade e com maior velocidade.

A manutenção do equilíbrio é considerada uma habilidade fundamental, pois constitui um pré-requisito para se iniciar qualquer movimento e depende de três sistemas aferentes que se intercorrelacionam entre si: somestésico, vestibular e visual. Diversas estratégias sensóriomotoras promovem estabilização do corpo e coordenam os movimentos oculares em relação ao meio ambiente. O equilíbrio é, portanto, uma capacidade coordenativa que promove estabilização estática e dinâmica ${ }^{20}$. O equilíbrio consiste na habilidade de manter o centro de massa do corpo dentro de determinada base de sustentaçáo em diferentes posições, o que requer estabilidade; envolve também transferir o peso do corpo delicadamente e precisamente dentro da base de sustentação em diferentes direções a partir do centro da base, onde se necessita ir até os limites da estabilidade, envolvendo orientação e alinhamento postural ${ }^{21}$.

Sendo assim, quaisquer alteraçóes de um dos sistemas e/ou do órgão efetor do movimento, por exemplo, um déficit no sistema somestésico, pode ser parcialmente compensado pelos demais sistemas sensoriais remanes- centes (vestibular ou visual), interferindo positivamente na antecipaçáo do movimento e no aprendizado motor ${ }^{20}$.

Interfere também no equilíbrio, o tono muscular que é regulado por estruturas que recebem informaçóes dos três sistemas, sendo que o sistema vestibular informa sobre modificações da posição da cabeça; o visual regula a percepção das relaçôes espaciais e o somestésico, através de receptores musculares, articulares, dos tendões, cápsulas articulares e da pele, informa sobre a postura e os deslocamentos das demais partes do corpo. Essas informaçóes extero e proprioceptivas auxiliam também na modulação do tono muscular necessário para manter a postura $^{20}$.

Os resultados obtidos na escala de marcha de Tinetti mostraram que houve uma melhora significativa após a intervenção fisioterapêutica sobre o trampolim, isto pode ter ocorrido não somente por interferência nas reaçóes de equilíbrio, mas em partes, por ter influenciado no tono muscular, provavelmente devido a hiperestimulação de vias aferentes, dentre elas a proprioceptiva.

As sensaçóes de pressão nos pés podem dizer se o peso está distribuído igualmente entre os dois pés e, se o mesmo está mais à frente ou atrás na base de sustentação. O movimento da lona sob os pés durante os exercícios pode ter facilitado a percepção ponderal e, consequentemente, influenciado positivamente na marcha, no entanto, para a paciente deambular sobre a superfície instável foi necessário também aumentar a consciência de seus demais segmentos corporais, utilizando reflexos contra a gravidade, já que o desequilíbrio provocado pela lona, tende a ser estímulo para manutenção do equilíbrio, ao mesmo tempo em que gera um trabalho constante de contraçóes excêntricas, principalmente dos músculos dos membros inferiores para se manter sobre a base própria de sustentação, o que provavelmente leva a uma inibição do reflexo miotático e, consequentemente ajuda na adequação do tono muscular ${ }^{22}$.

O escore de Tinetti, no follow-up houve piora do resultado, sendo que o item comprimento e altura do passo $(4-2)$ e tronco $(2-1)$ tiveram redução da pontuação. Isto nos permite inferir que o tratamento no trampolim colaborou para melhora da marcha dessa paciente imediatamente após o término do tratamento, no entanto, no follow up de 6 meses, sem tratamento, não houve 
Tabela 3

Valores obtidos antes, imediatamente após e, após 6 meses (follow up) do tratamento, utilizando como instrumento de mensuração a escala de Ashwort

\begin{tabular}{|c|c|c|c|c|c|c|}
\hline \multirow[t]{2}{*}{$\begin{array}{l}\text { Articulaçáo/ } \\
\text { Movimento }\end{array}$} & \multicolumn{2}{|c|}{$\begin{array}{c}\text { Avaliaçáo } \\
\text { Pré Tratamento }\end{array}$} & \multicolumn{2}{|c|}{$\begin{array}{c}\text { Avaliaçáo } \\
\text { Pós Tratamento }\end{array}$} & \multicolumn{2}{|c|}{ Follow $U_{p}$} \\
\hline & D & $\mathbf{E}$ & D & $\mathbf{E}$ & D & $\mathbf{E}$ \\
\hline \multicolumn{7}{|l|}{ Ombro } \\
\hline Flexão & $\mathbf{0}$ & $\mathbf{0}$ & $\mathbf{0}$ & $\mathbf{0}$ & $\mathbf{0}$ & $\mathbf{0}$ \\
\hline Extensão & $\mathbf{0}$ & $\mathbf{0}$ & $\mathbf{0}$ & $\mathbf{0}$ & $\mathbf{0}$ & $\mathbf{0}$ \\
\hline Abduçãoo & $\mathbf{0}$ & $\mathbf{0}$ & $\mathbf{0}$ & $\mathbf{0}$ & $\mathbf{0}$ & $\mathbf{0}$ \\
\hline Adução & $\mathbf{0}$ & $\mathbf{0}$ & $\mathbf{0}$ & $\mathbf{0}$ & $\mathbf{0}$ & $\mathbf{0}$ \\
\hline Rotação Lateral & $\mathbf{0}$ & $\mathbf{0}$ & $\mathbf{0}$ & $\mathbf{0}$ & $\mathbf{0}$ & $\mathbf{0}$ \\
\hline Rotação Medial & $\mathbf{0}$ & $\mathbf{0}$ & $\mathbf{0}$ & $\mathbf{0}$ & $\mathbf{0}$ & $\mathbf{0}$ \\
\hline \multicolumn{7}{|l|}{ Cotovelo } \\
\hline Flexão & $\mathbf{0}$ & $\mathbf{0}$ & $\mathbf{0}$ & $\mathbf{0}$ & $\mathbf{0}$ & $\mathbf{0}$ \\
\hline Extensão & $\mathbf{0}$ & $\mathbf{0}$ & $\mathbf{0}$ & $\mathbf{0}$ & $\mathbf{0}$ & $\mathbf{0}$ \\
\hline Pronação & $\mathbf{0}$ & $\mathbf{0}$ & $\mathbf{0}$ & $\mathbf{0}$ & $1 \#$ & $\mathbf{0}$ \\
\hline Supinação & $\mathbf{0}$ & $\mathbf{0}$ & $\mathbf{0}$ & $\mathbf{0}$ & $\mathbf{0}$ & $\mathbf{0}$ \\
\hline \multicolumn{7}{|l|}{ Punho } \\
\hline Flexão & $\mathbf{0}$ & $\mathbf{0}$ & $1 \#$ & $\mathbf{0}$ & $\mathbf{0}^{*}$ & $\mathbf{0}$ \\
\hline Extensão & $\mathbf{0}$ & $\mathbf{0}$ & $\mathbf{0}$ & $\mathbf{0}$ & $1 \#$ & $\mathbf{0}$ \\
\hline Desvio Radial & $\mathbf{0}$ & $\mathbf{0}$ & $\mathbf{0}$ & $\mathbf{0}$ & $1 \#$ & $\mathbf{0}$ \\
\hline Desvio Ulnar & $\mathbf{0}$ & $\mathbf{0}$ & $\mathbf{0}$ & $1 \#$ & $\mathbf{0}$ & $\mathbf{0}^{*}$ \\
\hline \multicolumn{7}{|c|}{$\operatorname{Dedos}\left(2^{\circ}\right.$. ao $\left.5^{\circ}.\right)$} \\
\hline Flexão & $\mathbf{0}$ & $\mathbf{0}$ & $\mathbf{0}$ & $\mathbf{0}$ & $\mathbf{0}$ & $\mathbf{0}$ \\
\hline Extensão & 4 & 3 & $3^{*}$ & $2^{*}$ & $1+^{*}$ & $1^{*}$ \\
\hline Abduçãoo & $\mathbf{0}$ & $\mathbf{0}$ & $\mathbf{0}$ & $\mathbf{0}$ & $\mathbf{0}$ & $\mathbf{0}$ \\
\hline Adução & $\mathbf{0}$ & $\mathbf{0}$ & $\mathbf{0}$ & $\mathbf{0}$ & $\mathbf{0}$ & $\mathbf{0}$ \\
\hline \multicolumn{7}{|l|}{ Quadril } \\
\hline Flexão & $1+$ & $\mathbf{0}$ & $1+$ & $\mathbf{0}$ & $1^{*}$ & $\mathbf{0}$ \\
\hline Extensão & 2 & $\mathbf{0}$ & $\mathbf{0}^{*}$ & $\mathbf{0}$ & $\mathbf{0}$ & $\mathbf{0}$ \\
\hline Abdução & 3 & 1 & $2^{*}$ & 1 & $1+^{*}$ & 1 \\
\hline Adução & $\mathbf{0}$ & $\mathbf{0}$ & $\mathbf{0}$ & $\mathbf{0}$ & $\mathbf{0}$ & $\mathbf{0}$ \\
\hline Rotação Lateral & 2 & 1 & $1^{*}$ & 1 & 1 & 1 \\
\hline Rotação Medial & 3 & $\mathbf{0}$ & $\mathbf{0}^{*}$ & $\mathbf{0}$ & $\mathbf{0}$ & $\mathbf{0}$ \\
\hline \multicolumn{7}{|l|}{ Joelho } \\
\hline Flexão & 1 & $\mathbf{0}$ & 1 & $\mathbf{0}$ & $\mathbf{0}^{*}$ & $\mathbf{0}$ \\
\hline Extensão & 4 & $1+$ & $2^{*}$ & $1^{*}$ & 2 & $\mathbf{0}^{*}$ \\
\hline \multicolumn{7}{|l|}{ Pé } \\
\hline Flexão Plantar & $\mathbf{0}$ & $\mathbf{0}$ & $\mathbf{0}$ & $\mathbf{0}$ & $\mathbf{0}$ & $\mathbf{0}$ \\
\hline Dorsoflexão & 4 & 3 & 4 & 3 & $3^{*}$ & $1+^{*}$ \\
\hline Inversão & $\mathbf{0}$ & 0 & $\mathbf{0}$ & 0 & $1 \#$ & 0 \\
\hline Eversão & $\mathbf{0}$ & $\mathbf{0}$ & $1+\#$ & $\mathbf{0}$ & $\mathbf{0}^{*}$ & $\mathbf{0}$ \\
\hline
\end{tabular}

Os valores com asterisco $\left(^{*}\right)$ indicam que houve alteração positiva e com jogo da velha (\#) negativa entre as avaliaçóes inicial, final e ou follow-up; legenda: $\mathrm{D}=$ direito, $\mathrm{E}=$ esquerdo 
Tabela 4

Valores obtidos antes, imediatamente após e, após 6 meses (follow up) do tratamento, utilizando a avaliação da flexibilidade articular com fleximêtro

\begin{tabular}{|c|c|c|c|}
\hline Segmento e Movimento & $\begin{array}{c}\text { Avaliaçáo } \\
\text { Pré Tratamento }\end{array}$ & $\begin{array}{c}\text { Avaliaçáo } \\
\text { Pós Tratamento }\end{array}$ & Follow $U_{p}$ \\
\hline \multicolumn{4}{|l|}{ Membro Superior Direito } \\
\hline Flexão de ombro & $151^{\circ}$ & $180^{\circ} *$ & $173^{\circ} \#$ \\
\hline Extensão de ombro & $40^{\circ}$ & $48^{\circ *}$ & $48^{\circ}$ \\
\hline Rotação medial de ombro & $42^{\circ}$ & $57^{\circ} *$ & $57^{\circ}$ \\
\hline Rotação lateral de ombro & $54^{\circ}$ & $76^{\circ} *$ & $67^{\circ} \#$ \\
\hline Abdução de ombro & $103^{\circ}$ & $114^{\circ *}$ & $156^{\circ} *$ \\
\hline Adução de ombro & $25^{\circ}$ & $25^{\circ}$ & $42^{\circ *}$ \\
\hline Flexão de cotovelo & $118^{\circ}$ & $118^{\circ}$ & $115^{\circ} \#$ \\
\hline Pronaçá̃o & $45^{\circ}$ & $80^{\circ} *$ & $90^{\circ} *$ \\
\hline Supinaçấo & $80^{\circ}$ & $80^{\circ}$ & $80^{\circ}$ \\
\hline Flexão de punho & $34^{\circ}$ & $43^{\circ *}$ & $57^{\circ} *$ \\
\hline Extensão de punho & $32^{\circ}$ & $32^{\circ}$ & $42^{\circ *}$ \\
\hline Desvio Ulnar & $34^{\circ}$ & $34^{\circ}$ & $23^{\circ} \#$ \\
\hline Desvio radial & $32^{\circ}$ & $22^{\circ} \#$ & $22^{\circ}$ \\
\hline \multicolumn{4}{|l|}{ Membro Superior Esquerdo } \\
\hline Flexão de ombro & $173^{\circ}$ & $128^{\circ} \#$ & $154^{\circ} *$ \\
\hline Extensão de ombro & $55^{\circ}$ & $63^{\circ *}$ & $51^{\circ} \#$ \\
\hline Rotação medial de ombro & $60^{\circ}$ & $60^{\circ}$ & $48^{\circ} \#$ \\
\hline Rotaçâo lateral de ombro & $80^{\circ}$ & $80^{\circ}$ & $62^{\circ} \#$ \\
\hline Abdução de ombro & $100^{\circ}$ & $115^{\circ *}$ & $128^{\circ *}$ \\
\hline Adução de ombro & $18^{\circ}$ & $31^{\circ} *$ & $31^{\circ}$ \\
\hline Flexão de cotovelo & $111^{\circ}$ & $126^{\circ *}$ & $136^{\circ} *$ \\
\hline Pronaçấo & $87^{\circ}$ & $80^{\circ} \#$ & $90^{\circ} *$ \\
\hline Supinação & $80^{\circ}$ & $80^{\circ}$ & $90^{\circ} *$ \\
\hline Flexão de punho & $45^{\circ}$ & $45^{\circ}$ & $51^{\circ *}$ \\
\hline Extensão de punho & $30^{\circ}$ & $30^{\circ}$ & $52^{\circ *}$ \\
\hline Desvio ulnar de punho & $29^{\circ}$ & $29^{\circ}$ & $23^{\circ} \#$ \\
\hline Desvio radial de punho & $30^{\circ}$ & $30^{\circ}$ & $37^{\circ *}$ \\
\hline \multicolumn{4}{|l|}{ Membro Inferior Direito } \\
\hline Flexão de quadril & $78^{\circ}$ & $78^{\circ}$ & $78^{\circ}$ \\
\hline Extensão de quadril & $15^{\circ}$ & $23^{\circ} *$ & $23^{\circ}$ \\
\hline Rotação medial de quadril & $19^{\circ}$ & $38^{\circ} *$ & $38^{\circ}$ \\
\hline Rotação lateral de quadril & $23^{\circ}$ & $46^{\circ} *$ & $46^{\circ}$ \\
\hline Abdução de quadril & $30^{\circ}$ & $24^{\circ} \#$ & $24^{\circ}$ \\
\hline Adução de quadril & $12^{\circ}$ & $12^{\circ}$ & $12^{\circ}$ \\
\hline Flexão de joelho & $110^{\circ}$ & $124^{\circ *}$ & $111^{\circ} \#$ \\
\hline Dorsiflexẫo de tornozelo & $6^{\circ}$ & $6^{\circ}$ & $11^{\circ *}$ \\
\hline Plantiflexão de tornozelo & $8^{\circ}$ & $8^{\circ}$ & $8^{\circ}$ \\
\hline Inversão do pé & $23^{\circ}$ & $23^{\circ}$ & $23^{\circ}$ \\
\hline Eversão do pé & $9^{\circ}$ & $16^{\circ} *$ & $16^{\circ}$ \\
\hline
\end{tabular}

Os valores com asterisco $\left({ }^{*}\right)$ indicam que houve alteração positiva e com jogo da velha (\#) negativa entre as avaliaçôes inicial, final e ou follow-up. 
Tabela 4

(Continuação)

\begin{tabular}{|c|c|c|c|}
\hline Segmento e Movimento & $\begin{array}{c}\text { Avaliaçáo } \\
\text { Pré Tratamento }\end{array}$ & $\begin{array}{c}\text { Avaliaçáo } \\
\text { Pós Tratamento }\end{array}$ & Follow $U_{p}$ \\
\hline \multicolumn{4}{|l|}{ Membro Inferior Esquerdo } \\
\hline Flexão de quadril & $110^{\circ}$ & $100^{\circ} \#$ & $100^{\circ}$ \\
\hline Extensão quadril & $20^{\circ}$ & $20^{\circ}$ & $20^{\circ}$ \\
\hline Rotação medial de quadril & $23^{\circ}$ & $37^{\circ *}$ & $27^{\circ}$ \\
\hline Rotação lateral de quadril & $22^{\circ}$ & $32^{\circ} *$ & $37^{\circ *}$ \\
\hline Abdução de quadril & $24^{\circ}$ & $33^{\circ *}$ & $33^{\circ}$ \\
\hline Adução Quadril & $17^{\circ}$ & $17^{\circ}$ & $17^{\circ}$ \\
\hline Flexão de joelho & $103^{\circ}$ & $118^{\circ *}$ & $118^{\circ}$ \\
\hline Dorsiflexão de tornozelo & $10^{\circ}$ & $20^{\circ} *$ & $28^{\circ *}$ \\
\hline Plantiflexão de tornozelo & $10^{\circ}$ & $22^{\circ} *$ & $17^{\circ} \#$ \\
\hline Inversão do pé & $23^{\circ}$ & $23^{\circ}$ & $23^{\circ}$ \\
\hline Eversão do pé & $14^{\circ}$ & $14^{\circ}$ & $26^{\circ} *$ \\
\hline
\end{tabular}

Os valores com asterisco $\left(^{*}\right)$ indicam que houve alteraçăo positiva e com jogo da velha (\#) negativa entre as avaliaçóes inicial, final e ou follow-up.

manutenção dos benefícios, o que sugere que a continuidade da terapêutica é necessária para a manutenção dos benefícios da técnica.

$\mathrm{Na}$ Ashworth houve aumento do tônus (espasticidade) de alguns movimentos de membros superiores, imediatamente após o término do tratamento. A espasticidade é definida como uma resposta velocidade-dependente do músculo à mobilização passiva. Estes autores descrevem que a espasticidade está entre as mais comuns sequelas reconhecidas do sistema nervoso central ${ }^{18}$. Embora seu papel real na disfunção motora possa ser menor do que sugerido por outros autores, os músculos antagonistas espásticos podem limitar a força gerada pelo músculo agonista durante um movimento voluntário de grande complexidade, como por exemplo, em situaçôes em que a instabilidade da superfície é grande. Esta pode ser uma hipótese para explicar este aumento do tono dos membros superiores.

Uma sugestão é a inclusão de estratégias específicas para membros superiores, assim como foi desenvolvido para os membros inferiores, no programa de tratamento com trampolim, por exemplo, incluir exercícios de cadeia cinética fechada para esses segmentos, envolvendo atividades que estimulem os estabilizadores das cinturas escapulares.
No programa cinesioterapêutico desse estudo, conforme o tratamento progredia, a realização de alguns exercícios, que já tinham sido treinados desde o começo tornava-se de execução mais fáceis, como, por exemplo, as transferências de peso ântero-posterior e látero-lateral sobre os pés, isso ocorre devido ao aprendizado motor, uma vez adquirida a experiência, a prática repetitiva leva a formação de perceptos cinestésicos e, essa memória passa a ser evocada para a execução da tarefa de maneira automática. No entanto, é claro que a paciente no início sentia-se insegura, pois era uma atividade nova, porém com o decorrer do tratamento, a repetição dos exercícios e, consequentemente o aprendizado motor, tornaram a tarefa fácil de ser executada. À medida que a paciente evoluía novos desafios eram incluídos, com exercícios de maior complexidade, o que levou a uma adequação individualizada do programa às necessidades da paciente.

Em relação aos membros inferiores houve redução da hipertonia muscular na maioria dos movimentos, exceto na eversão de MID e inversão de MIE, uma justificativa para tal, pode ser que a paciente sobre uma superfície instável, devido já apresentar uma marcha patológica pregressa (em tesoura com excessiva rotação medial e adução de quadris, eversão do tornozelo direito e inversão do tornozelo esquerdo), utilizou-se da exacerbação do padrão 
na tentativa de manter-se estável dentro de sua própria base de sustentação.

Em relação à flexibilidade articular a paciente apresentou melhora na maioria dos movimentos articulares imediatamente após a intervenção fisioterapêutica, sendo que algumas destas ainda mantiveram-se e, outras regrediram seis meses após o término do tratamento (follow-up). Isto nos reporta a imaginar que exercícios aplicados sobre o trampolim podem ter sido facilitadores para melhora da mobilidade geral da paciente, principalmente no dimídio direito, hemicorpo, inicialmente mais acometido. $\mathrm{O}$ trampolim por ser uma superfície instável parece proporcionar maior mobilidade articular, o que consequentemente interferiu positivamente na flexibilidade articular. A mobilização articular aumenta a atividade biológica, movimenta mais o líquido sinovial, trás mais nutrientes para a cartilagem, facilita sua lubrificação e, consequentemente propicia aumento da flexibilidade articular ${ }^{23}$.

No entanto, em algumas articulações, imediatamente após o tratamento houve movimentos que pioraram a amplitude de movimento. Isso também pode ser justificado pelo aumento do padrão patológico por meio de fixaçôes de alguns segmentos articulares mais acometidos, quando submetidos a situaçôes de instabilidade postural, na tentativa de estabilizá-los, gerando bloqueio da movimentação ativa e diminuição da amplitude do movimento, interferindo negativamente na flexibilidade articular.

Como demonstrado nesse estudo, essa nova estratégia fisioterapêutica teve efeitos positivos nesse caso, no entanto, os autores desse estudo sugerem que esse programa deve ser aprimorado na tentativa de minimizar os efeitos negativos, e testado em outros processos nosológicos do sistema nervoso, em um número maior de pacientes, e em tempos de duração do programa diferentes a fim de analisar seus efeitos em diferentes situaçôes.

\section{CONCLUSÃO}

Este trabalho mostrou que a intervenção fisioterapêutica, cinesioterapêutica, utilizando o recurso trampolim, melhorou o equilíbrio durante a marcha imediatamente após o tratamento, no entanto, após seis meses do término do tratamento, os benefícios em relação ao equilíbrio durante a marcha não se mantiveram.
Verificou-se também que houve influência desta intervenção em relação ao tono muscular e a flexibilidade articular, ora positiva ora negativamente, e após seis meses do término do tratamento a manutenção desses itens foi variável.

Concluindo-se, portanto que a cinesioterapia com o trampolim, em um caso de traumatismo raquimedular incompleto nível C7-T1, teve efeitos positivos em relação ao equilíbrio, marcha, em algumas articulaçôes no concernente a flexibilidade articular e em alguns músculos em relação ao tono muscular. No entanto, a manutenção destes benefícios após seis meses do término do tratamento variou.

\section{REFERÊNCIAS}

1.Dawodu ST. Spinal cord injury: definition, epidemiology, pathophysiology. Rehab Sports Med Assoc: Emedicine J 2001;2:307-21.

2.Dumont RJ, Okonkwo DO, Verma S, Hurlbert RJ, Boulos PT, Ellegala DB, et al. Acute spinal cord injury, part I: Pathophysiologic Mechanisms. Clin Neuropharmacol 2001;24:254-64.

http://dx.doi.org/10.1097/00002826-200109000-00003

http://dx.doi.org/10.1097/00002826-200109000-00002

3.Marotta JT. Traumatismo Raquimedular. In: Rowland LP. Merrit Tratado de Neurologia. 9a. ed. Rio de Janeiro: Guanabara Koogan, 1997, p.350-4.

4.Masini M. Estimativa da incidência e prevalência de lesão medular no Brasil. J. Bras. Neurocirurg. 2001;12:97-100.

5.Mederdrut EM, Araújo MLM. Trampolim Terapêutico [apostilado]. São Paulo: Centro de Performance Humana; 2004, p.3-5.

6.Otsuka GH, Fontes SV, Mederdrut EM, Fukujima MM. Effects of physiotherapy using the therapeutic springboard in balance of in ataxic patient. Arq Neuropsiquiatr 2004;62(supl2):77.

7.Garcia T. Efeitos da cinesioterapia utilizando como recurso coadjuvante da cinesioterapia o trampolim terapêutico no equilíbrio, na flexibilidade, no tônus muscular e na marcha de um paciente com esclerose múltipla (Trabalho de Conclusão de Curso). São Bernardo do Campo: Umesp, 2004, 119p.

8.Consani T. Efeitos da cinesioterapia utilizando como recurso o trampolim terapêutico no equilíbrio e coordenaçáo motora de paciente com paralisia cerebral (Trabalho de Conclusão de Curso). São Bernardo do Campo: Umesp, 2004, 62p.

9.Zanelli S. Efeitos da intervenção fisioterapêutica utilizando como recurso coadjuvante da cinesioterapia o trampolim terapêutico na flexibilidade articular, no equilíbrio, na marcha e na qualidade de vida de paciente com doença de Parkinson (Trabalho de Conclusão de Curso). São Bernardo do Campo: UMESP, 2004, 141p.

10.Krebs PW. Efeitos da cinesioterapia utilizando o recurso trampolim terapêutico no equilíbrio e na marcha de paciente com acidente vascular encefálico (Trabalho de Conclusão de Curso). São Bernardo do Campo: UMESP, 2004, 84p.

11.Barak A, Wexler ID, Efrati O, Bentur L, Augarten A, Mussaffi H, et al. 
Trampoline use as Physiotherapy for Cystic Fibrosis Patients. Pediatr Pulmonol 2005;39:70-3.

http://dx.doi.org/10.1002/ppul.20133

12.Tiburcio TT. Revisão bibliográfica de instrumentos utilizados para avaliação do equilíbrio em indivíduos adultos, (Trabalho de Conclusão de Curso). São Bernardo do Campo: Umesp, 2004, 80p.

13.Berg KO, Maki BE, Williams JI, Holliday PJ, Wood-dauphinee SL. Clini$\mathrm{cal}$ and laboratory measures of postural balance in an elderly population. Arch Phys Med Rehabil 1992;73:1073-82.

14.Berg KO, Wood-Dauphinee SL, Williams JI, Maki BE. Measuring Bce in the Elderly: Validation of an instrument. Can J Public Health 1992;83:7-11.

15.Tinetti B, Raiche M, Hebert R, Prince F, Corriveau H. Screening older adults at risk of falling with the Tinetti Balance Scale. Lancet 2000;356:1001-2. http://dx.doi.org/10.1016/S0140-6736(00)02695-7

16.Shumway-Cook A, Horak FB, Yardley L, Bronstein AM. Rehabilitation of Balance Disorders in the Patient with Vestibular Pathology. In: Bronstein AM, Brandt T, Woollacott MH. Clinical disorders of balance, posture and gait. London: Arnold; 1996, p.213-4.
17.Bohannon RW, Smith MB. Interrater Reliaability of a Modified Ashworth Scales of Muscles Spasticity. Phys Ther 1987;67:206-7.

18.Achour JA. Avaliando a flexibilidade: fleximeter. Londrina: Midiograf,. Manual de instruçōes, 1997, 79p.

19.Fontes SV, Alves MAF, Ottoboni C, Fukujima MM. Classificação dos procedimentos fisioterápicos. In: Fontes SV, Fukujima MM, Cardeal JO. Fisioterapia Neurofuncional: fundamentos para a prática. São Paulo: Atheneu, 2007, p.149-64.

20.Moreira MMF, Maudonnet OAQ. Equilíbrio: conceitos básicos e mecanismos visuais no controle do equilíbrio. Acta Awho 1998;17:66-9.

21.Ragnarsdottir M. The concept of balance. Physiother 1996;82:368-75.

http://dx.doi.org/10.1016/S0031-9406(05)66484-X

22.Guyton AC, Hall JE. Tratado de Fisiologia Médica. 10ª ed. Rio de Janeiro: Guanabara Koogan, 2002, 973p.

23. Kisner C, Colby LA. Amplitude de movimento. In: Kisner C, Colby LA. Exercícios terapêuticos: fundamentos e técnicas. $4^{a}$.ed. São Paulo: Manole, 2005, 841p. 